\title{
A Materials Acceleration Platform for Organic Laser Discovery
}

Tony C Wu ${ }^{1, *}$, Andrés Aguilar Granda ${ }^{1, *,}$, Kazuhiro Hotta ${ }^{1,5}$, Sahar Alasvand Yazdani ${ }^{2}$, Robert Pollice ${ }^{1}$, Jenya Vestfrid ${ }^{1}$, Han Hao ${ }^{1}$, Cyrille Lavigne ${ }^{1}$, Martin Seifrid ${ }^{1}$, Nicholas Angello $^{3}$, Fatima Bencheikh², Jason E. Hein ${ }^{4}$, Martin Burke ${ }^{3}$, Chihaya Adachi ${ }^{2}$, Alán Aspuru-Guzik ${ }^{1, \dagger}$

1. Department of Chemistry, University of Toronto, Toronto, ON M5S 3H6, Canada

2. Center for Organic Photonics and Electronics Research, Kyushu University, Fukuoka 819-0395, Japan

3. Department of Chemistry, University of Illinois at Urbana-Champaign, Urbana, IL 61801, USA

4. Department of Chemistry, University of British Columbia, Vancouver, BC V6T 1Z1, Canada

5. Science \& Innovation Center, Mitsubishi Chemical Corporation, Yokohama, 227-8502, Japan

" equal contributions. ${ }^{\dagger}$ email: aspuru@utoronto.ca. ${ }^{\ddagger}$ present address: Facultad de Química, Universidad Nacional Autónoma de México, México.

\section{Abstract:}

Conventional materials discovery is a laborious and time-consuming process that can take decades from initial conception of the material to commercialization. Recent developments in materials acceleration platforms promise to accelerate materials discovery using automation of experiments coupled with machine learning. However, most of the automation efforts in chemistry focus on synthesis and compound identification, with integrated target property characterization receiving less attention. In this work, we introduce an automated platform for the discovery of molecules as gain mediums for organic semiconductor lasers, a problem that has been challenging for conventional approaches. Our platform encompassed automated lego-like synthesis, product identification, and optical characterization that can be executed in a fully integrated end-to-end fashion. Using this workflow to screen organic laser candidates, we have discovered 8 potential candidates for organic lasers. We tested the lasing threshold of 4 molecules in thin-film devices and found 2 molecules with state-of-the-art performance. These promising results show the potential of automated synthesis and screening for accelerated materials development. 


\section{Introduction}

Lasers have unique light-emitting properties such as coherence, directionality, monochromaticity, and high intensity. Consequently, lasers have become important in many fields, such as communication, data storage, medicine, and industrial manufacturing. A key component of a laser device is the gain medium. Solid-state gain mediums benefit from their compact size, ease of operation and high intensity. Until recently, solid-state lasers typically employ inorganic materials. Considerable research efforts have been directed at the development of organic semiconductor lasers (OSL). OSLs offer significant potential for color tunability, low-cost fabrication, and mechanical flexibility ${ }^{1-4}$. For instance, various classes of organic semiconductors ${ }^{5}$ exhibit high optical gain, thus enabling their application as organic lasers and optical amplifiers. However, further breakthroughs in the development of organic molecules as a laser gain medium are still needed for continuous-wave operation and electrically pumped devices $^{6-8}$.

Finding appropriate organic molecules for OSLs has proven to be challenging ${ }^{9,10}$ due to thermal degradation ${ }^{11}$ and gain losses from triplet excitons ${ }^{12,13}$ or charge carriers. While traditional materials discovery is a slow process, recent developments in self-driving labs can accelerate the optimization process dramatically by combining automated experimental systems with data-driven workflows. Along with the machine learning revolution, research groups and industries have been developing better and more reliable automated systems for use in the fields of chemistry ${ }^{14-20}$, biotechnology $y^{21-23}$ and electronics ${ }^{24,25}$. For example, $\mathrm{Li}$ et al ${ }^{26}$ developed an automated and modular building block-based synthesis platform based on iterative Suzuki-Miyaura cross-coupling reactions. This synthetic strategy utilizes an iterative deprotection, coupling, and purification sequence for repeated additions of MIDA boronate-functionalized building blocks, creating complex functional molecules via a lego-like approach. However, most automated systems for chemistry have been developed for synthesis and compound identification, but rarely end-to-end integration with target property characterization.

In this work, we developed an end-to-end automated platform to screen organic molecules as potential laser gain mediums. Our platform encompasses organic synthesis, product identification, and optical characterizations of potential laser gain mediums, wherein all parts run automatically from start to finish. The target molecular space in our screening consisted of 40 candidates, which were derived from the state-of-the-art laser molecule 4,4'-Bis[(N-carbazole)styryl]biphenyl (BSBCz $)^{27-29}$. 


\section{End-to-end Automated Screening Platform}

Our organic laser screening platform consists of three main parts, all of which are automated: (i) organic synthesis, (ii) product purification and identification, and (iii) optical characterization. An overview of our platform is provided as an action flow chart in Figure 1a. Apart from preparing the starting materials, the platform is fully automated without manual interruption.

The candidate laser molecules were synthesized in the Chemspeed platform. After the synthesis, the obtained reaction mixtures were diluted and automatically transferred to our high-performance liquid chromatography mass spectrometry (HPLC-MS) equipment through the Chemspeed injection valves for subsequent purification and product identification. With the target molecules identified, the Chemspeed injected the mixture for a second time to the HPLC-MS and the purified materials were transferred to our optical characterization setup based on their retention time.

The optical characterization setup was custom built for our organic laser property screening. Using the optical flow cells, we measured the absorption and emission spectra, as well as transient emission from the molecules. Based on these results, the cross-section gains, $\sigma_{\text {gain }}$ (Equation S1), were estimated to assess whether the candidates might be appropriate laser gain materials.

All the automated steps were controlled through separate Python programs. To manage the synchronization between all the programs such as transferring materials from the Chemspeed platform to the HPLC-MS and diverting the product stream to optical characterization setup, the programs on different computers exchange communication files through Dropbox. Additional details are provided in Section S1.8 and Figure S9.

\section{Organic Laser Molecule Synthesis}

The synthesis of $\mathrm{BSBC} z$ has been reported by others ${ }^{30}$ through a Buchwald-Hartwig cross-coupling followed by a Witting-Horner reaction. Although the reaction yields are good, the purification steps on these synthesis sequences can be a limiting factor to the generation of molecular libraries to molecules with similar optical properties quickly. As an alternative, iterative Suzuki couplings were selected to be able to automate the synthesis $^{26}$ of $\mathrm{BSBC} z$ derivatives in a single step. The intermediate carbazolyl styryl-BMIDA 1a was synthesized manually in large scale by Suzuki-Miyaura cross-coupling (Figure 2a), where the carbazolyl phenyl boronic acid pinacol ester was coupled with trans-2-bromovinylboronic acid MIDA ester in good yield. In the automated step performed using the Chemspeed platform, the target BSBCz derivatives were synthesized employing a one-pot in situ deprotection and Suzuki-Miyaura cross-coupling reaction sequence. This one-pot strategy reduces the time and 
resources required for work-up and purification making it highly amenable to automation. This approach also obviates isolating the labile vinylboronic acid intermediate derived from 1a. The 40 dihalide starting materials used to synthesize the $\mathrm{BSBC} z$ derivatives are depicted in Figure $2 \mathrm{~b}$. The selection of the dihalides was based on our goal to investigate a large variety of aromatic rings and functional groups, as well as on considerations of commercial availability and pricing. The BSBCz derivatives resulting from the selected dihalides are shown in Figure S3. Notably, the original $\mathrm{BSBC} z$ molecule was also included in our target compounds so that we could directly compare the optical characterization results of the novel molecules to it.

\section{Organic Laser Molecule Screening}

The $40 \mathrm{BSBC} z$ derivatives were synthesized and tested using the autonomous screening workflow. An example video of our automated reactions can be viewed in Video S1. Overall, the entire automated process took almost 2 days, requiring approximately 20 hours for synthesis and 24 hours for characterization. Across the 40 syntheses carried out, 33 yielded the corresponding products as confirmed via HPLC-MS. The remaining 7 products could not be detected, most likely due to either low product yields or low solubilities of the corresponding products in the solvents used (tetrahydrofuran for synthesis and acetonitrile for HPLC-MS). For all the molecules detected, our platform automatically recorded chromatograms, mass spectra, absorption spectra, emission spectra, excitation (UV) absorptions, and transient emissions. From these results, we determined relative PLQYs, emission rates, and the relative maximum cross-section gains, $\sigma_{\text {gain }}$, of the molecules. All the optical parameters are summarized in Table S1.

For good organic lasers, we need to find molecules that maximize the stimulated emission cross-section gain, $\sigma_{\text {gain }}$. Using our automated optical characterization setup, we approximated this figure of merit based on the emission spectra, relative PLQYs, and emission rates measured in acetonitrile solutions. Figure $3 a$ shows the cross-section gains and maximum cross-section gain wavelengths of the 33 successfully identified molecules. The cross-section gain of $B S B C z$ (1c), which to date is believed to be the state-of-the-art organic laser molecule, is labeled in green. Notably, in the top left corner of the plot, there are 8 molecules labeled red that we found to have larger cross-section gains than BSBCz. Taking a closer look into one of our best-performing candidates, 5c (Figure 3b), the corresponding optical absorption and emission spectra are superimposed in Figure $3 \mathrm{c}$ and the emission lifetime data is depicted in Figure 3d. Additionally, in Figure S18, we plotted the relative PLQY values against the emission lifetimes of the molecules. All the 8 top-performing molecules have both high relative PLQYs and short emission lifetimes $(<1.4 \mathrm{~ns})$, similar to BSBCz. This 
is consistent with our target to maximize cross-section gains, which mainly relies on both high PLQYs and fast emission rates.

In addition to fully automated synthesis and optical characterization, we performed quantum chemical simulations to gain valuable insight into the structure-property relationships of the 33 synthesized and characterized products. We simulated the optical spectra accounting for Franck-Condon factors via a path integral method at the B3LYP/6-31G* level of theory ${ }^{31,32}$. The comparison of the simulated and experimental first absorption and emission peaks are depicted in Figure 4a and Figure S19. We observed excellent agreement for the absorption spectra and a systematic offset but still an acceptable correlation for the emission spectra. This shows that our computations properly reflect the experimental optical properties of these compounds.

Since direct simulation of laser performance parameters was challenging, we looked for other decisive computed parameters to achieve high cross-section gains, which could help us establish principles for designing better laser molecules. Indeed, we identified some properties that might be required for high cross-section gains as illustrated in Figure 4c-e. Importantly, none of these properties alone seem to be sufficient to effect high performance which points towards dependencies on multiple factors and causes direct correlations to be small. Future work is necessary to verify these design leads.

When decomposing the candidates into cores, including the vinyl moieties, and donor fragments, as illustrated in Figure $4 \mathrm{~b}$, and investigating the corresponding highest occupied molecular orbital (HOMO) energies, we found the molecules with the highest cross-section gains to have a core HOMO energy that equals the HOMO energy of the donor (Figure 4c). This suggests that a donor-acceptor-donor design scheme, which is prevalent in other organic electronic materials like organic light-emitting diodes ${ }^{33-36}$, is not effective in organic lasers, and rather points towards adopting a molecular design strategy where the core and donor units share similar HOMO levels. Nevertheless, matching the HOMO levels alone does not suffice for a good laser candidate as can be seen in molecule 23c (cf. Table S2). Whereas corresponding HOMO levels of the core and donor unit are essentially identical (within less than $0.001 \mathrm{eV}$ ), the cross-section gain is only intermediate $\left(0.10 \times 10^{-15} \mathrm{~cm}^{-2}\right)$. However, the decreased cross-section gain could be caused by the free aromatic amine. Hence, the analysis of more derivatives is required to understand these trends better.

Additionally, we observed that the oscillator strength between the first excited singlet state $S_{1}$ and the ground state $S_{0}$ is high for all the candidates with high cross-section gains (Figure S20). This is expected as the oscillator strength influences the fluorescence rate, which in turn affects the cross-section gain (Equation S1). However, our data also shows that a high oscillator strength alone is insufficient for good organic 
lasers, as demonstrated for instance by molecule 34c. It has one of the highest oscillator strengths (3.86) but one of the lowest cross-section gains $\left(0.02 \times 10^{-15} \mathrm{~cm}^{-2}\right)$.

Finally, looking into excited state descriptors based on the physics of correlated electron-hole pairs ${ }^{37}$, we found that the candidates with the highest cross-section gains have a linear electron-hole separation of close to zero (Figure 4d). Due to the symmetric donor-core-donor construction, values close to zero indicate that the corresponding structures have a linear 3-dimensional geometry with the two donor units spatially as far apart as possible. Hence, a linear 3-dimensional arrangement seems favorable for good organic lasers. Additionally, candidates with high cross-section gains have a large root-mean-square electron-hole separation (Figure S21). This not only shows that the linear electron-hole separation is close to zero due to symmetry but also indicates a significant separation between electron and hole. This was corroborated by the charge-transfer numbers which indicate a mixed locally excited and charge-transfer character in the corresponding transitions. The locally excited character stems from local excitations situated on the donor fragment. The charge-transfer character stems from significant contributions from a charge-resonance state, i.e., simultaneous charge-transfer excitations from the donor to the core and vice versa. Importantly, we found this charge-resonance character to be high in the best organic laser candidates (Figure 4e). Hence, we hypothesize that the design of molecules with significant charge-resonance character is relevant to achieve good organic lasers. Further theoretical and experimental studies are required to build a better understanding of this observed structure-property trend. Overall, every top-performing molecule in this work has matching HOMO levels between core and donor units, high oscillator strength for the transition between the ground state and the first excited singlet state, and a high charge-resonance character in the corresponding excited state.

\section{Lasing in Thin-Film Devices}

Four of the top-performing molecules (Figure S22) were selected for further investigations in thin films. The molecules were doped in a host of 4,4'-Bis(N-carbazolyl)-1,1'-biphenyl (CBP), which was fabricated by thermal co-deposition. The corresponding optical measurements we performed include determinations of PLQY, emission rate, and amplified spontaneous emission (ASE) threshold which is a direct measure to test whether a molecule is suitable for organic lasers.

From the thin film results, $\mathbf{3 c}$ and $\mathbf{5 c}$ are the most promising organic laser candidates with low ASE thresholds of 1.2 and $1.4 \mu \mathrm{J} / \mathrm{cm}^{2}$, respectively. These ASE thresholds are comparable to that of BSBCz at $1.1 \mu \mathrm{J} / \mathrm{cm}^{2}$. The input-output characteristics that show the lasing thresholds of $\mathbf{3 c}$ and $\mathbf{5 c}$ are depicted in Figure $5 a-b$, with the other measured 
results shown in Figure S23. The emission spectra at different excitation energies are shown Figure $5 \mathrm{c}-\mathrm{d}$. Additionally, detailed thin-film optical properties of the molecules, such as PLQYs, emission rates, ASE thresholds, and emission cross-section gains are summarized in Table S3.

\section{Conclusions and Outlook}

High-throughput virtual screening and machine learning are already revolutionizing chemistry, and materials acceleration platforms represent the next frontier for accelerating materials development. We have built an end-to-end automated screening platform encompassing organic synthesis, product identification and optical characterization to identify promising laser candidates. We have demonstrated the capabilities of our platform by both synthesizing and characterizing various $B S B C z$ derivatives in our system. We synthesized the molecules in our Chemspeed synthesis robot. Then, we used HPLC-MS for purifying and identifying our target products, which allowed us to perform online product analysis, streamlining our workflow significantly. Finally, our custom-built optical characterization setup enables immediate measurement of important optical properties. While conventional approaches require manual synthesis, purification and characterization using a number of different tools, our system performs all these steps in a single, fully automated workflow. Since it requires minimal manual intervention, it frees up researchers time for higher level tasks and can be integrated with high-throughput virtual screening or machine learning approaches.

The automated screening allowed us to identify 8 laser candidates with larger cross-section gains than BSBCz. Among these candidates, we tested the lasing threshold of 4 molecules in thin-film devices, and found 2 molecules with state-of-the-art performances. These promising results demonstrated the potential of automated screening for accelerated materials development. Further expansion to a larger screening space and the use of machine learning to propose candidates could potentially find even better organic laser molecules. This work has been started and is currently under development.

Our organic laser screening platform synthesized and characterized 33 molecules under identical conditions providing valuable insight to understand the underlying structure-property relationships of good laser molecules. Based on our simulations, we suggest that matching the HOMO level of the donor with the HOMO level of the core is beneficial. Furthermore, a larger oscillator strength and linear molecular geometries may be important to achieve high cross-section gains. Finally, we found that substantial charge-resonance contributions might be essential for good organic lasers. Nevertheless, future screening of more diverse structures and additional characterization methods is essential to establish them as robust design parameters. 
Overall, this work is a demonstration of the substantial potential of end-to-end automated systems to accelerate materials discovery. Our platform can readily be expanded to screen organic molecules for applications such as OLEDs, organic solar cells, and organic redox flow batteries. We plan to expand our automated workflow further and integrate machine learning with quantum chemistry simulations directly into the platform to enable genuine computer-driven closed-loop materials discovery. 


\section{Figures}

(a)



(b)

(c)

(d)


Figure 1. Synthesis workflow and implementation of the experimental platform. (a) Schematic representation of automated workflow for screening organic molecules. (i) synthesis in Chemspeed, (ii) identification and separation in HPLC-MS, and (iii) optical property characterization in custom built setup. (b) Top-down view of the Chemspeed deck, which includes ISYNTH (vortex mixing, heating, reflux), vial racks, and overhead liquid handler. (c) Front view of the automated system of identification and characterization of target molecules (HPLC-MS). (d) Custom built optical measurement. Here the product (in solution) is transferred through a flow cuvette (bottom left corner). The excitation is from a PicoQuant laser diode (LDH series; 100 picoseconds) and the transient photoluminescence intensity is measured by time-correlated single photon counting from PicoQuant TimeHarp 260. 
(a)
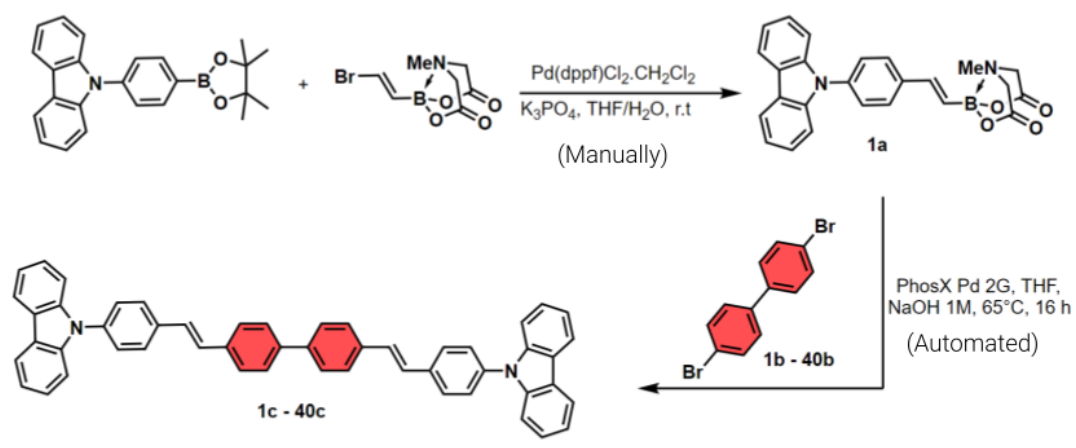

(b)



Figure 2. One-pot Suzuki cross-coupling applied in the automated synthesis in the Chemspeed. (a) Generic scheme for the cross-coupling synthesis of BSBCz using a MIDA boronate and 4,4' dibromobiphenyl core. (b) Commercially accessible dihalide cores selected as molecular scaffolds in the synthesis of BSBCz derivatives. 




(b) Top screening molecule: $\mathbf{5 c}$

(c)
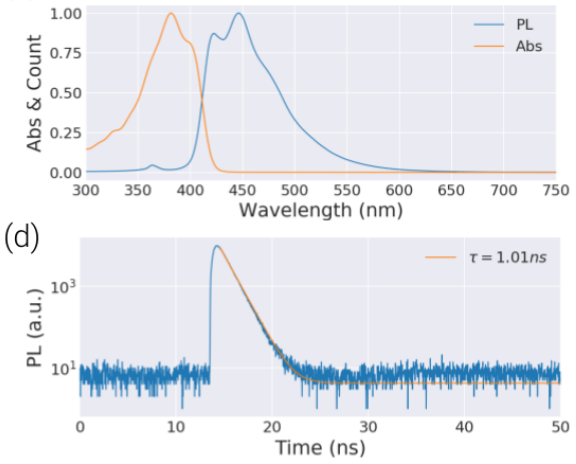

Figure 3. (a) Measured peak cross-section gains compared to their corresponding wavelengths. 33 out of 40 samples are successfully synthesized, isolated, and measured. 8 samples (labeled in red) are the potential laser candidates that demonstrate a larger cross-section gain than BSBCz (labeled green). (b) Example of the top organic laser molecule candidate 5c. (c) The photoluminescence spectra and absorption spectra of molecule 5c. It has a peak emission at $445 \mathrm{~nm}$. (d) The transient emission of molecule 5c. The orange line is the fitted exponential decay, with $1.01 \mathrm{~ns}$ of emission lifetime. 


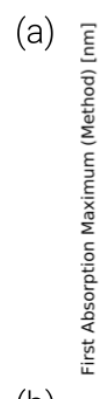

(b)



(c)
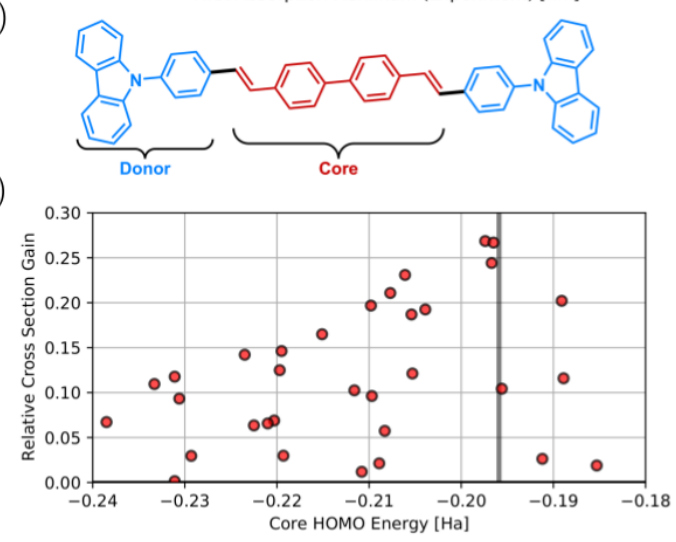

(d)



(e)

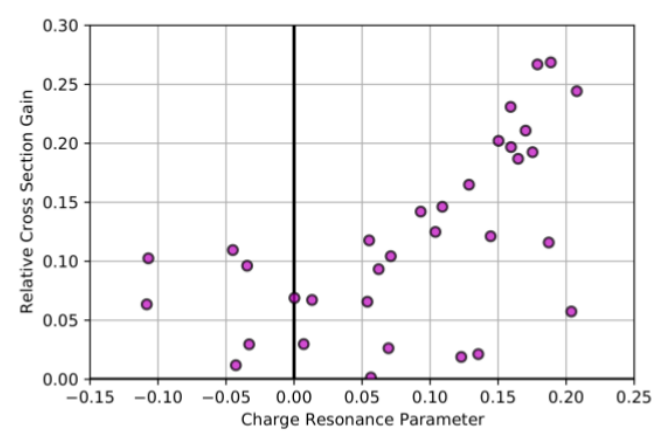

Figure 4. (a) Comparison of experimental first absorption maxima against simulated first absorption maxima. The solid line indicates what would be a perfect simulation that matches the experimental data exactly. (b) Donor and core of BSBCz-derivatives used in theoretical studies. (c) Scatter plot of relative cross-section gain against the HOMO energy of the core fragment capped with hydrogen atoms. The vertical gray line indicates the HOMO energy of the donor fragment capped with hydrogen. (d) Scatter plot of relative cross-section gain against the linear electron-hole separation in the first excited singlet state. (e) Scatter plot of relative cross-section gain against the charge-resonance parameter in the first excited singlet state. 

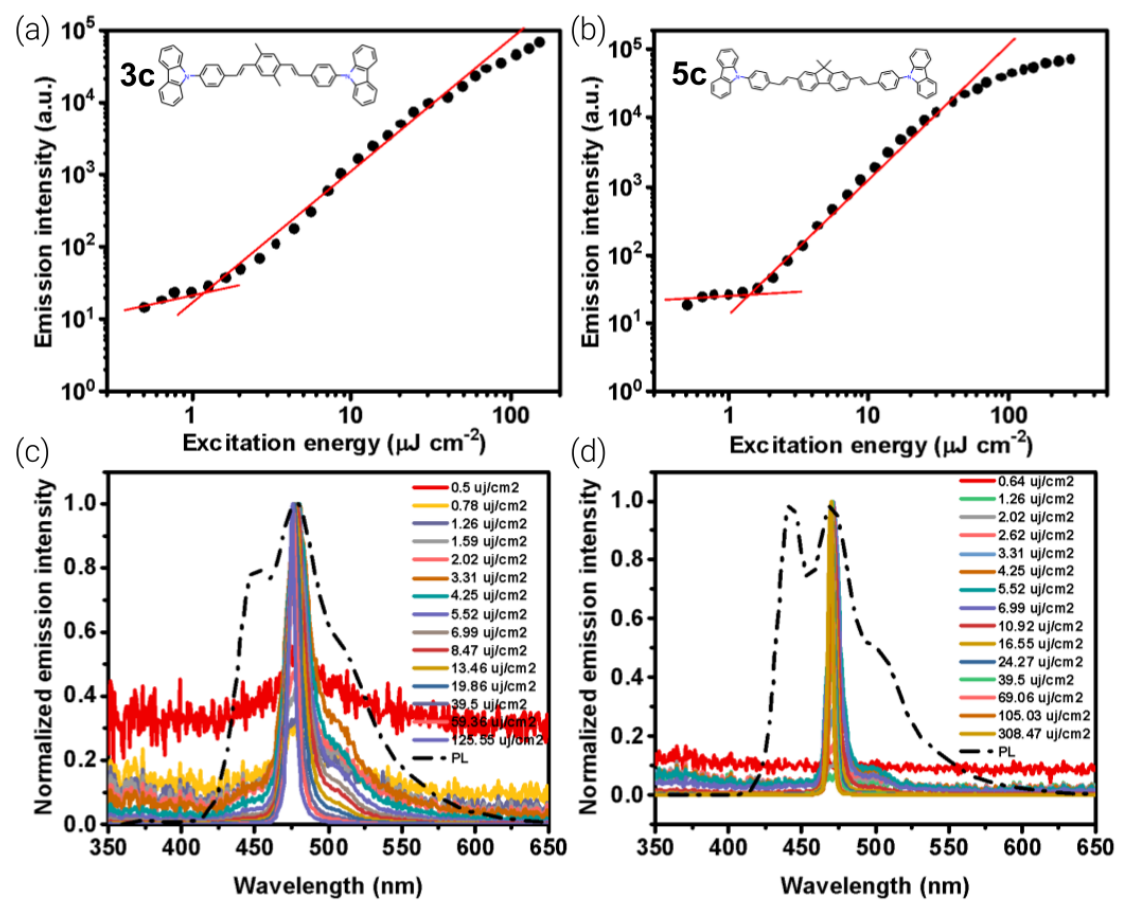

Figure 5. The amplified spontaneous emission (ASE) threshold of thin-film devices for $3 c$ and $\mathbf{5 c}$ molecules. (a-b) Emission intensity of $\mathbf{3 c}$ and $\mathbf{5 c}$ against excitation energy. (c-d) Emission spectra of $\mathbf{3 c}$ and $\mathbf{5 c}$ under different excitation energies are shown. 


\section{Methods}

\section{Automated Synthesis in Chemspeed}

The coupling for synthesizing the BSBCz derivatives (Figure 2a) was automated in our customized ISYNTH Chemspeed platform. Details of the synthesis are provided in Section S1.2 and S1.5. First, we manually prepared stock solutions of all substrates, i.e., intermediate $\mathbf{1 b}$, dihalide, catalyst and base. The resulting solutions were placed on the vial racks of the Chemspeed platform. All subsequent steps were executed automatically without human intervention. First, the 4-needle liquid handler distributed the appropriate amount of each reagent into the ISYNTH reactor, which was then sealed for the reaction. The reactor was heated to $65^{\circ} \mathrm{C}$ and agitated using a vortex at $400 \mathrm{rpm}$. After 16 hours of reaction, the reactor was cooled down to room temperature and the agitation was turned off allowing the reaction mixtures to both cool down and settle.

To characterize the products, the 4-needle liquid handler of the Chemspeed platform sampled the organic phase of the reaction mixtures and diluted the samples 10 -fold in separate vials using tetrahydrofuran. The solutions were homogenized by repeated rounds of draw and dispense operations. After dilution, the samples were injected into a Chemspeed injection valve, and then transferred to the HPLC-MS.

\section{Separation and Identification in HPLC-MS}

All the product mixtures were separated and identified using an HPLC-MS (ThermoFisher, Vanquish and Q Exactive). When the injection valve from Chemspeed switches position, it triggers an electrical signal which starts a predefined HPLC-MS method that uses acetonitrile/water solvent mixtures (see Section S1.6). The following analyses were used to detect the target $\mathrm{BSBC} z$ derivatives and obtain their retention times. All target compound peaks were identified using selected ion chromatograms (XIC) based on the masses of the molecular ions or their respective protonated/deprotonated species. Chemical identities were further confirmed by comparing predicted against observed isotope patterns for the molecular ions in the mass spectra. After successful confirmation of peak identities, the corresponding retention times were recorded.

To estimate the concentrations of the BSBCz derivatives suitable for subsequent optical characterizations, we used the 3D chromatograms obtained from the HPLC diode array detector (DAD). First, the target peak in the DAD chromatogram was located using the retention time determined as described above using MS. Then, the absorbances at the wavelength of the absorption maximum and at the wavelength for subsequent PL excitation (i.e. $365 \mathrm{~nm}$ ) were calculated for the target peak. This information was used to estimate the required injection sample concentration for the optical characterization. 
Then, the 4-needle liquid handler samples more reaction mixture and transfers it to the HPLC-MS for a second separation. Using the retention time from the first injection, the target compounds eluting from the HPLC-MS are redirected selectively into collection vials using a flow selector. These collected samples are then used for the optical characterization.

\section{Custom-built automated optical characterization}

Using a syringe pump, the collected samples are transferred into an additional vial for dilution with ACN. Subsequently, the diluted samples are transferred to flow cuvettes (Helma flow-through cuvettes) for all the optical property measurements. Detailed descriptions of the characterizations are provided in Section S1.7.

For the absorption spectra, we used a white light lamp (Ocean Insight, DH-MINI) and a spectrometer (Ocean Insight, QEPro XR), which was also used for measuring the emission spectra. This setup can measure the absorption spectra from 260 to $980 \mathrm{~nm}$. For characterizing photoluminescence, we used a $365 \mathrm{~nm}$ solid-state LED (Thorlabs, M365FP1 with DC4100HUB) for the excitation and a photodetector (Thorlabs, S120VC with PM100D) for measuring the excitation transmission. From the integration of the emission spectrum and the absorption of the excitation source, we could calculate the relative photoluminescence quantum yield (PLQY) of the sample.

To measure the transient emission of the sample, we used a picosecond laser (PicoQuant, LDH-D-C-375 with PDL800-D) for excitation and an avalanche photodiode (Micro photon device, PDM) for detection. The transient emission statistics were collected by a time-correlated single photon counting (TCSPC) board (PicoQuant, TimeHarp 260). The transient emission optical measurement had a minimal detection limit of 200 picoseconds, which is suitable for the range of emission rates in the BSBCz derivatives.

\section{Calculations and Simulations of Optical Properties}

Ground state conformational ensembles were generated using the CREST computer package $^{38}$ (version 2.11.1) with the iMTD-GC ${ }^{39,40}$ workflow (default option) at the GFN2-xTB ${ }^{41-43}$ level of theory. The lowest energy conformers were first reoptimized using $\mathrm{xtb}^{44}$ (version 6.4.1) at the GFN2-xTB level of theory, followed by another reoptimization using Q-Chem ${ }^{45}$ (version 5.4) at the B3LYP ${ }^{46-48} / 6-31 G^{* 49-51}$ level of theory. Absorption and emission spectra and fluorescence rate calculations were performed using Franck-Condon calculations via a gradient-based method, which was described previously ${ }^{31,52}$, at the previously benchmarked ${ }^{53,54}$ B3LYP/6-31G* level of theory using Q-Chem ${ }^{45}$ (version 5.4). Both experimental and simulated spectra were evaluated using the standard signal processing algorithms implemented in scipy.signal for peak detection ${ }^{55}$. HOMO and LUMO orbital energies were taken 
directly from ground-state single point calculations at the B3LYP/6-31G* level of theory of the optimized geometries. Exciton parameters were simulated using TheoDORE ${ }^{56,57}$ (version 2.0.2) based on the Q-Chem outputs of the B3LYP/6-31G* excited state calculations of the full organic laser candidates. More details of the corresponding simulations are provided in Section S4.1.

\section{Device Fabrication and Characterization}

To fabricate the films for photo-physical measurements, the quartz and glass substrates were cleaned by sonication with alkali detergent, pure water, Acetone, Isopropanol (IPA) subsequently for 10 minutes each, followed by UV-ozone treatments for 15 minutes. Next, the thin-film devices were vacuum deposited at the pressure of $\sim 10^{-5} \mathrm{~Pa}$ with $6 \%$ weight of laser molecules doped in CBP for $200 \mathrm{~nm}$. The absorption spectra were measured using a UV-VIS spectrometer (LAMBDA950-PKA PerkinElmer) and the photoluminescence spectra were obtained using a spectrofluorometer (FP-8600 Jasco). In addition, the PLQYs and the emission lifetimes of the laser molecules were measured with a PLQY spectrometer (HAMAMATSU C11347) and a fluorescence lifetime spectrometer (HAMAMATSU C11367), respectively. These photo-physical measurements were conducted under air.

The ASE thresholds of the organic laser molecules were measured by optical excitation of films with a nitrogen gas laser (Stanford Research Systems NL100) at an excitation wavelength of $337 \mathrm{~nm}$ with a $3 \mathrm{~ns}$ pulse width and pulse frequency of $20 \mathrm{~Hz}$. The input excitation laser beam was focused through a cylindrical lens into a stripe resulting in a $0.43 \mathrm{~cm} \times 0.11 \mathrm{~cm}$ beam area. All ASE measurements were performed under nitrogen atmosphere to prevent degradation. Output light emission was detected with a spectrometer (Hamamatsu Photonics PMA-12), where the emission was collected from the edge of the sample with an optical fiber. Finally, the ASE thresholds were obtained from the input-output intensity characteristic figures.

\section{Acknowledgements}

We acknowledge the Defense Advanced Research Projects Agency (DARPA) under the Accelerated Molecular Discovery Program under Cooperative Agreement No. HR00111920027 dated August 1, 2019. The content of the information presented in this work does not necessarily reflect the position or the policy of the Government. All computations reported in this work were performed on the Niagara supercomputer at the SciNet HPC Consortium. SciNet is funded by the Canada Foundation for Innovation, the Government of Ontario, Ontario Research Fund-Research Excellence, and by the University of Toronto. T.C.W. acknowledges funding through University of Toronto Arts \& Science Postdoctoral Fellowship. R.P. acknowledges funding through a 
Postdoc.Mobility fellowship by the Swiss National Science Foundation (SNSF, Project No. 191127). We acknowledge important discussions with Rafael Gómez-Bombarelli (MIT) and Stéphane Kéna Cohen (Polytechnique Montréal). A.A.G. acknowledges generous support from Anders G. Frøseth. We acknowledge equipment support from the Canadian Foundation for Innovation and the Ontario Research Fund as well as support from the Canada 150 Research Chairs Program. We acknowledge Lumtec Inc. for custom synthesis of candidate materials.

\section{Author contributions}

(Alán Aspuru-Guzik as AAG, Andrés Aguilar Granda as A-AG) AAG, TCW, and A-AG designed the research. TWC and A-AG contributed equally to this work. TCW, A-AG and $\mathrm{JV}$ designed the synthesis in Chemspeed and programmed the controls. $\mathrm{KH}$ programmed the controls of HPLC-MS. TCW and KH built and programmed the controls for the optical measurements. A-AG and TCW ran the screening experiments. NA provided suggestions for the synthesis and helped with the methodology. RP and $C L$ performed the theoretical studies. HH, A-AG, RP and TCW analyzed the measurement of the NMR and HRMS for the selected molecules. MS helped develop HPLC recipes. SAY fabricated and measured the devices. AAG, MB and JEH supervised chemistry automation. AAG supervised the computational chemistry study. CA and FB supervised the measured device fabrication. All authors contributed to writing and editing the manuscript.

\section{References}

1. Clark, J. \& Lanzani, G. Organic photonics for communications. Nature Photonics vol. 4 438-446 (2010).

2. Moses, D. High quantum efficiency luminescence from a conducting polymer in solution: A novel polymer laser dye. Applied Physics Letters vol. 60 3215-3216 (1992).

3. Samuel, I. D. W. \& Turnbull, G. A. Organic semiconductor lasers. Chem. Rev. 107, 1272-1295 (2007).

4. Chénais, S. \& Forget, S. Recent advances in solid-state organic lasers. Polymer International vol. 61 390-406 (2012).

5. Kuehne, A. J. C. \& Gather, M. C. Organic Lasers: Recent Developments on Materials, 
Device Geometries, and Fabrication Techniques. Chem. Rev. 116, 12823-12864 (2016).

6. Samuel, I. D. W., Namdas, E. B. \& Turnbull, G. A. How to recognize lasing. Nature Photonics vol. 3 546-549 (2009).

7. Bisri, S. Z., Takenobu, T. \& Iwasa, Y. The pursuit of electrically-driven organic semiconductor lasers. J. Mater. Chem. 2, (2014).

8. Sandanayaka, A. S. D. et al. Indication of current-injection lasing from an organic semiconductor. Appl. Phys. Express 12, 061010 (2019).

9. Bornemann, R., Thiel, E. \& Bolívar, P. H. High-power solid-state cw dye laser. Opt. Express 19, 26382-26393 (2011).

10. Bornemann, R., Lemmer, U. \& Thiel, E. Continuous-wave solid-state dye laser. Opt. Lett. 31, 1669-1671 (2006).

11. Zhao, Z., Mhibik, O., Leang, T., Forget, S. \& Chénais, S. Thermal effects in thin-film organic solid-state lasers. Opt. Express 22, 30092-30107 (2014).

12. Baldo, M. A., Holmes, R. J. \& Forrest, S. R. Prospects for electrically pumped organic lasers. Physical Review B vol. 66 (2002).

13. Lehnhardt, M., Riedl, T., Weimann, T. \& Kowalsky, W. Impact of triplet absorption and triplet-singlet annihilation on the dynamics of optically pumped organic solid-state lasers. Physical Review B vol. 81 (2010).

14. Coley, C. W. et al. A robotic platform for flow synthesis of organic compounds informed by Al planning. Science 365, (2019).

15. Sun, S. et al. Accelerated Development of Perovskite-Inspired Materials via High-Throughput Synthesis and Machine-Learning Diagnosis. Joule 3, 1437-1451 (2019).

16. Burger, B. et al. A mobile robotic chemist. Nature 583, 237-241 (2020).

17. Chatterjee, S., Guidi, M., Seeberger, P. H. \& Gilmore, K. Automated radial synthesis of organic molecules. Nature 579, 379-384 (2020).

18. Collins, N. et al. Fully Automated Chemical Synthesis: Toward the Universal Synthesizer. 
Organic Process Research \& Development vol. 24 2064-2077 (2020).

19. MacLeod, B. P. et al. Self-driving laboratory for accelerated discovery of thin-film materials. Sci Adv 6, eaaz8867 (2020).

20. Christensen, M. et al. Data-science driven autonomous process optimization. Communications Chemistry 4, 1-12 (2021).

21. King, R. D. et al. Functional genomic hypothesis generation and experimentation by a robot scientist. Nature 427, 247-252 (2004).

22. Hoffer, L. et al. Integrated Strategy for Lead Optimization Based on Fragment Growing: The Diversity-Oriented-Target-Focused-Synthesis Approach. J. Med. Chem. 61, 5719-5732 (2018).

23. Chow, S., Liver, S. \& Nelson, A. Streamlining bioactive molecular discovery through integration and automation. Nature Reviews Chemistry 2, 174-183 (2018).

24. Chung, S.-L. \& Jeng, M. An overview of semiconductor fab automation systems. in 2003 IEEE International Conference on Robotics and Automation (Cat. No.03CH37422) vol. 1 1050-1055 vol.1 (2003).

25. Lee, T.-E. Semiconductor Manufacturing Automation. in Springer Handbook of Automation (ed. Nof, S. Y.) 911-926 (Springer Berlin Heidelberg, 2009).

26. Li, J. et al. Synthesis of many different types of organic small molecules using one automated process. Science 347, 1221-1226 (2015).

27. Aimono, T. et al. $100 \%$ fluorescence efficiency of 4,4 [sup ']-bis[(N-carbazole)styryl]biphenyl in a solid film and the very low amplified spontaneous emission threshold. Applied Physics Letters vol. 86071110 (2005).

28. Nakanotani, H., Adachi, C., Watanabe, S. \& Katoh, R. Spectrally narrow emission from organic films under continuous-wave excitation. Applied Physics Letters vol. 90231109 (2007).

29. Sandanayaka, A. S. D. et al. Toward continuous-wave operation of organic semiconductor 
lasers. Sci Adv 3, e1602570 (2017).

30. Mamada, M., Fukunaga, T., Bencheikh, F., Sandanayaka, A. S. D. \& Adachi, C. Low Amplified Spontaneous Emission Threshold from Organic Dyes Based on Bis-stilbene. Advanced Functional Materials vol. 281802130 (2018).

31. Baiardi, A., Bloino, J. \& Barone, V. General Time Dependent Approach to Vibronic Spectroscopy Including Franck-Condon, Herzberg-Teller, and Duschinsky Effects. J. Chem. Theory Comput. 9, 4097-4115 (2013).

32. de Souza, B., Neese, F. \& Izsák, R. On the theoretical prediction of fluorescence rates from first principles using the path integral approach. J. Chem. Phys. 148, 034104 (2018).

33. Im, Y. et al. Molecular Design Strategy of Organic Thermally Activated Delayed Fluorescence Emitters. Chem. Mater. 29, 1946-1963 (2017).

34. Konidena, R. K. \& Lee, J. Y. Molecular Design Tactics for Highly Efficient Thermally Activated Delayed Fluorescence Emitters for Organic Light Emitting Diodes. Chem. Rec. 19, 1499-1517 (2019).

35. Wong, M. Y. \& Zysman-Colman, E. Purely Organic Thermally Activated Delayed Fluorescence Materials for Organic Light-Emitting Diodes. Adv. Mater. 29, (2017).

36. Liu, Y., Li, C., Ren, Z., Yan, S. \& Bryce, M. R. All-organic thermally activated delayed fluorescence materials for organic light-emitting diodes. Nature Reviews Materials 3, 1-20 (2018).

37. Kimber, P. \& Plasser, F. Toward an understanding of electronic excitation energies beyond the molecular orbital picture. Phys. Chem. Chem. Phys. 22, 6058-6080 (2020).

38. crest: Conformer-Rotamer Ensemble Sampling Tool based on the xtb Semiempirical Extended Tight-Binding Program Package. (Github).

39. Grimme, S. Exploration of Chemical Compound, Conformer, and Reaction Space with Meta-Dynamics Simulations Based on Tight-Binding Quantum Chemical Calculations. J. Chem. Theory Comput. 15, 2847-2862 (2019). 
40. Pracht, P., Bohle, F. \& Grimme, S. Automated exploration of the low-energy chemical space with fast quantum chemical methods. Phys. Chem. Chem. Phys. 22, 7169-7192 (2020).

41. Grimme, S., Bannwarth, C. \& Shushkov, P. A Robust and Accurate Tight-Binding Quantum Chemical Method for Structures, Vibrational Frequencies, and Noncovalent Interactions of Large Molecular Systems Parametrized for All spd-Block Elements ( $Z=1-86)$. J. Chem. Theory Comput. 13, 1989-2009 (2017).

42. Bannwarth, C., Ehlert, S. \& Grimme, S. GFN2-xTB-An Accurate and Broadly Parametrized Self-Consistent Tight-Binding Quantum Chemical Method with Multipole Electrostatics and Density-Dependent Dispersion Contributions. J. Chem. Theory Comput. 15, 1652-1671 (2019).

43. Bannwarth, C. et al. Extended tight-binding quantum chemistry methods. Wiley Interdiscip. Rev. Comput. Mol. Sci. 11, (2021).

44. xtb: Semiempirical Extended Tight-Binding Program Package. (Github).

45. Shao, Y. et al. Advances in molecular quantum chemistry contained in the Q-Chem 4 program package. Mol. Phys. 113, 184-215 (2015).

46. Becke, A. D. Density-functional exchange-energy approximation with correct asymptotic behavior. Phys. Rev. A Gen. Phys. 38, 3098-3100 (1988).

47. Lee, C., Yang, W. \& Parr, R. G. Development of the Colle-Salvetti correlation-energy formula into a functional of the electron density. Phys. Rev. B Condens. Matter 37, 785-789 (1988).

48. Becke, A. D. Density-functional thermochemistry. III. The role of exact exchange. J. Chem. Phys. 98, 5648-5652 (1993).

49. Ditchfield, R., Hehre, W. J. \& Pople, J. A. Self-Consistent Molecular-Orbital Methods. IX. An Extended Gaussian-Type Basis for Molecular-Orbital Studies of Organic Molecules. J. Chem. Phys. 54, 724-728 (1971).

50. Hehre, W. J., Ditchfield, R. \& Pople, J. A. Self-Consistent Molecular Orbital Methods. XII. 
Further Extensions of Gaussian-Type Basis Sets for Use in Molecular Orbital Studies of Organic Molecules. J. Chem. Phys. 56, 2257-2261 (1972).

51. Hariharan, P. C. \& Pople, J. A. The influence of polarization functions on molecular orbital hydrogenation energies. Theor. Chim. Acta 28, 213-222 (1973).

52. de Souza, B., Neese, F. \& Izsák, R. On the theoretical prediction of fluorescence rates from first principles using the path integral approach. J. Chem. Phys. 148, 034104 (2018).

53. Charaf-Eddin, A., Planchat, A., Mennucci, B., Adamo, C. \& Jacquemin, D. Choosing a Functional for Computing Absorption and Fluorescence Band Shapes with TD-DFT. J. Chem. Theory Comput. 9, 2749-2760 (2013).

54. Humeniuk, A. et al. Predicting fluorescence quantum yields for molecules in solution: A critical assessment of the harmonic approximation and the choice of the lineshape function. J. Chem. Phys. 152, 054107 (2020).

55. Virtanen, P. et al. SciPy 1.0: fundamental algorithms for scientific computing in Python. Nat. Methods 17, 261-272 (2020).

56. Plasser, F. TheoDORE: A toolbox for a detailed and automated analysis of electronic excited state computations. J. Chem. Phys. 152, 084108 (2020).

57. TheoDORE. https://sourceforge.net/projects/theodore-qc/. 